\title{
Two more triangle centers
}

Mowaffaq Hajja and Panagiotis T. Krasopoulos

Mowaffaq Hajja received his Ph.D. at Purdue University (Indiana, USA) in 1978 Since then he holds a position at Yarmouk University in Irbid (Jordan) where he is currently a professor. His mathematical interests include field theory and Euclidean geometry.

Panagiotis T. Krasopoulos received his Ph.D. in Engineering from the National Technical University of Athens (NTUA), Greece, in 2001. Currently, he is working at the Social Insurance Institute as a computer scientist. His mathematical interests include geometry, dynamical systems, and optimization.

\section{Introduction}

This note grew out of a question that a student asked while the first-named author was giving a lecture on triangle centers in a Geometry class in the fall of 2008. The student, Shefa'a Bani Melhem, wondered about the point in a given triangle that, when joined to the midpoints of the sides, divides the triangle into three quadrilaterals of equal area. Few days later, the aforementioned author asked her to prove that such a point is necessarily the centroid and to consider the point whose perpendiculars to the sides divide the triangle into three quadrilaterals of equal area. This problem turned out to be more difficult than was expected, and it is the purpose of this note to investigate the existence and uniqueness of such a point and to describe its trilinear coordinates (or simply, its trilinears), i.e., its

In dem nachfolgenden Beitrag untersuchen die Autoren die naheliegende Fragestellung, ob es in einem spitzwinkligen Dreieck $A B C$ einen Punkt $\mathcal{E}$ mit der Eigenschaft gibt, dass die drei Vierecke, die durch das Fällen der Lote von $\mathcal{E}$ auf die Dreiecksseiten entstehen, flächengleich sind. Die Autoren beweisen, dass es in einem spitzwinkligen Dreieck genau einen solchen Punkt $\mathcal{E}$ gibt. Sie bestimmen auch die Gleichungen, denen die Abstände von $\mathcal{E}$ zu den Dreiecksseiten genügen. Im allgemeinen ist der Punkt $\mathcal{E}$ vom Umkreis- und Innkreismittelpunkt sowie vom Schwerpunkt und vom Höhenschnittpunkt verschieden. Fällt der Punkt $\mathcal{E}$ aber mit einem dieser klassischen Zentren zusammen, so weisen die Autoren nach, dass ein gleichseitiges Dreieck vorliegt. 
directed distances to the sides of the triangle. The question whether this center can coincide with any of the traditional centers for a non-equilateral triangle is also addressed. We call such a point the equiareality center and we denote it by $\mathcal{E}$.

We show that $\mathcal{E}$ exists and is unique for acute triangles, and we write down equations that define its trilinears. However, these equations, though quite simple looking and elegant, are very hard to solve and everything suggests that this center is new. We also prove that this new center does not coincide with any of the traditional centers except when the triangle is equilateral.

In the course of investigation, a natural question arose and led to another center that we denoted by $\mathcal{E}_{0}$. Its properties and relation to other centers are also explored.

Many of the items in this paper can be used as projects, homeworks, examination problems, and issues for classroom discussion in a first course in Euclidean Geometry. In particular, dealing with the complexities that arise when one considers obtuse triangles is expected to generate fruitful discussions and to lead to interesting results.

\section{Existence and uniqueness of $\mathcal{E}$ for acute triangles}

Theorem 3 below establishes the existence and uniqueness of $\mathcal{E}$ for acute triangles. Lemma 1 is needed in its proof. In this lemma and throughout, the side lengths and angles of a triangle $A B C$ are denoted by $a, b, c, A, B, C$, in the standard order, and the symbol $[\ldots]$ stands for the area.

Lemma 1. Let $A B C$ be an acute triangle and let $X$ be a point on the side $B C$. Let $X Y$, $X Z$ be the perpendiculars dropped from $X$ onto the sides $A C, A B$, respectively.

(i) If $[X Y C] \geq \frac{[A B C]}{4}$, then $[X Z B]<\frac{[A B C]}{4}$.

(ii) If $A \leq B$ and $A \leq C$, then $\frac{[X Z B]+[X Y C]}{[A B C]} \leq \frac{1}{2}$.

Proof. (i) Suppose that $[X Y C] \geq[A B C] / 4$. Let $X C=t$ and let $B S, C T$ be the perpendiculars dropped from $B, C$ on $A C, A B$, respectively; see Fig. 1. Then

$$
\frac{1}{4} \leq \frac{[X Y C]}{[A B C]}<\frac{[X Y C]}{[S B C]}=\frac{t^{2}}{a^{2}}
$$

Therefore $(t / a)^{2}>1 / 4$ and hence $t / a>1 / 2$ and $(a-t) / a<1 / 2$. Therefore

$$
\frac{[X Z B]}{[A B C]}<\frac{[X Z B]}{[T B C]}=\left(\frac{a-t}{a}\right)^{2}<\left(\frac{1}{2}\right)^{2}=\frac{1}{4}
$$

(ii) Let $t, S, T$ be as in (i) and refer to Fig. 1 again. Suppose that $A \leq B, A \leq C$. Then $[A B C] \geq 2[T B C],[A B C] \geq 2[S B C]$. It follows that

$$
\frac{[X Z B]}{[A B C]}+\frac{[X Y C]}{[A B C]} \leq \frac{[X Z B]}{2[T B C]}+\frac{[X Y C]}{2[S B C]}=\frac{t^{2}}{2 a^{2}}+\frac{(a-t)^{2}}{2 a^{2}} \leq \frac{a^{2}}{2 a^{2}}=\frac{1}{2},
$$

as desired. 


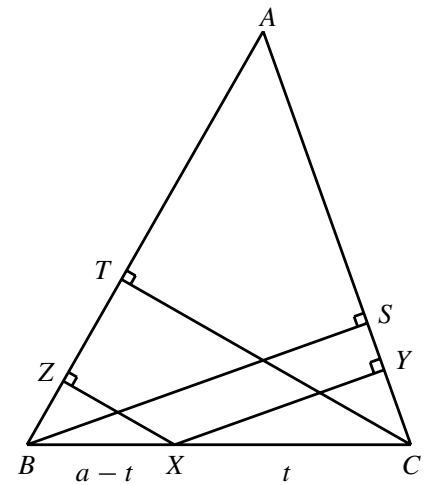

Fig. 1

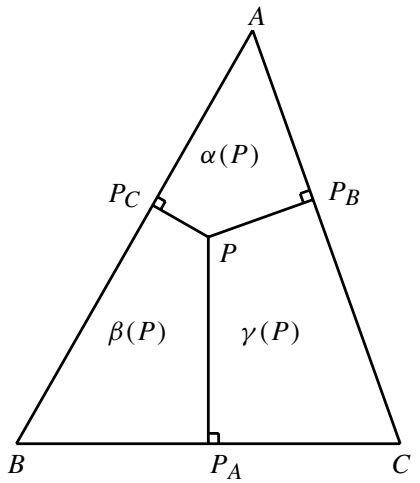

Fig. 2

We remark that an interesting elaboration on Lemma 1(ii) can be found in [3].

Before proving the main theorem, we introduce notations and definitions that we shall use throughout.

Definition 2. For any acute triangle $A B C$, and any point $P$ inside (or on the boundary of) $A B C$, we denote by $P_{A}, P_{B}, P_{C}$ the orthogonal projections of $P$ on the sides $B C, C A$, $A B$, respectively; see Fig. 2. We define $\alpha(P), \beta(P), \gamma(P)$ by

$$
\alpha(P)=\frac{\left[A P_{C} P P_{B}\right]}{[A B C]}, \quad \beta(P)=\frac{\left[B P_{A} P P_{C}\right]}{[A B C]}, \quad \gamma(P)=\frac{\left[C P_{B} P P_{A}\right]}{[A B C]} .
$$

Note that if $P$ is on the boundary, then some of these quadrilaterals degenerate into triangles.

Theorem 3. For any acute triangle, there exists a unique point whose perpendiculars to the sides divide the triangle into three quadrilaterals of equal area.

Proof. Let $A B C$ be an acute triangle, and assume without loss of generality that $A \leq B \leq C$.

Let $P$ be any point on the line segment $B C$; see Fig. 3. As a point $S$ moves from $A$ to $P, \alpha(S)$ increases from $\alpha(A)=0$ to $\alpha(P)$. By Lemma 1 (ii), $\alpha(P) \geq 1 / 2$. Therefore, there exists a unique point on $A P$ for which $\alpha=1 / 3$. We denote this point by $P^{*}$. Thus for every $P$ on $B C, P^{*}$ is the point on $A P$ for which $\alpha\left(P^{*}\right)=1 / 3$. In particular, $B^{*}$ is the point on $A B$ whose perpendicular $B^{*} U$ to $A C$ has the property that $\alpha\left(B^{*}\right)=$ $\left[B^{*} U A\right] /[A B C]=1 / 3$; see Fig. 4. Similarly, $C^{*}$ is the point on $A C$ whose perpendicular $C^{*} V$ to $A B$ has the property that $\alpha\left(C^{*}\right)=\left[C^{*} V A\right] /[A B C]=1 / 3$.

Since $\alpha\left(B^{*}\right)=1 / 3 \geq 1 / 4$, Lemma 1 (i) implies that $\beta\left(B^{*}\right)<1 / 4<1 / 3$. Similarly, $\gamma\left(C^{*}\right)<1 / 3$. Since $\alpha\left(B^{*}\right)=\alpha\left(C^{*}\right)=1 / 3$ and $\alpha+\beta+\gamma=1$, it follows that

$$
\beta\left(B^{*}\right)<\frac{1}{3}, \quad \beta\left(C^{*}\right)>\frac{1}{3} .
$$




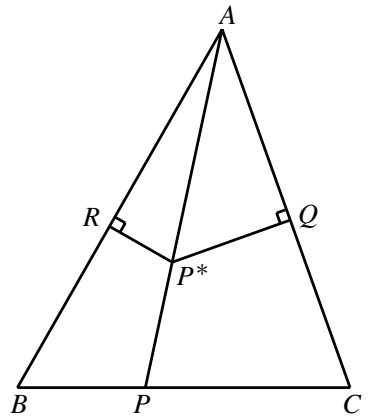

Fig. 3

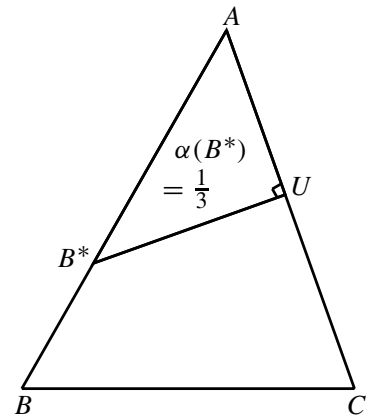

Fig. 4

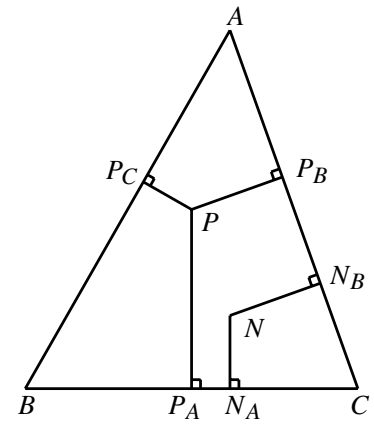

Fig. 5

By continuity of the function $P \mapsto \beta\left(P^{*}\right)$, there exists a point $M$ on $B C$ such that $\beta\left(M^{*}\right)=1 / 3$. Since $\alpha\left(M^{*}\right)=1 / 3$, it follows that $\alpha\left(M^{*}\right)=\beta\left(M^{*}\right)=\gamma\left(M^{*}\right)=1 / 3$, and hence $M^{*}$ has the required property.

To prove uniqueness, let $P, N$ be two points with the given property. Then $N$ must lie in one of the quadrilaterals determined by $P$, say $N$ lies in $C P_{B} P P_{A}$; see Fig. 5. Then $\gamma(N) \leq \gamma(P)$ with equality if and only if $N=P$. Since $\gamma(N)=\gamma(P)=1 / 3$, it follows that $N=P$.

Definition 4. For an acute triangle $A B C$, the point $P$ for which

$$
\alpha(P)=\beta(P)=\gamma(P)
$$

is called the equiareality center of $A B C$ and is denoted by $\mathcal{E}$.

\section{Trilinears of $\mathcal{E}$}

Let $A B C$ be an acute triangle and let $x, y, z$ be the trilinears of the equiareality center $\mathcal{E}$. Drop perpendiculars $\mathcal{E} Y, \mathcal{E} Z$ onto the sides $A C, A B$, respectively. Let $A=2 \xi, B=2 \eta$, $C=2 \zeta$. Let $\angle B A \mathcal{E}=\xi-p, \angle C A \mathcal{E}=\xi+p$; see Fig. 6 . Then

$$
\frac{z}{y}=\frac{\sin (\xi-p)}{\sin (\xi+p)}=\frac{\sin \xi \cos p-\cos \xi \sin p}{\sin \xi \cos p+\cos \xi \sin p}=\frac{1-\cot \xi \tan p}{1+\cot \xi \tan p}
$$

$$
\begin{aligned}
\cot \xi \tan p & =\frac{y-z}{y+z}, \\
\tan p & =\frac{y-z}{y+z} \tan \xi .
\end{aligned}
$$




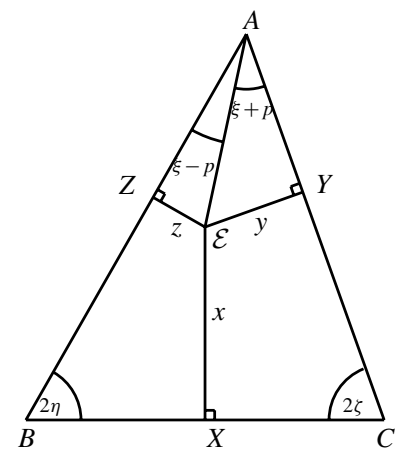

Fig. 6

$$
\begin{aligned}
2[A Z \mathcal{E} Y] & =y z \sin (2 \xi)+y \cot (\xi+p) z \cot (\xi-p) \sin 2 \xi \\
& =y z \sin (2 \xi)(1+\cot (\xi+p) \cot (\xi-p)) \\
& =y z \sin (2 \xi)\left(1+\frac{1-\tan \xi \tan p}{\tan \xi+\tan p} \frac{1+\tan \xi \tan p}{\tan \xi-\tan p}\right) \\
& =y z \sin (2 \xi) \frac{\left(1+\tan ^{2} \xi\right)\left(1-\tan ^{2} p\right)}{\tan ^{2} \xi-\tan ^{2} p} \\
& =2 y z \sin \xi \cos \xi \frac{\sec ^{2} \xi\left(1-\tan ^{2} p\right)}{\tan ^{2} \xi-\tan ^{2} p} \\
& =2 y z \tan \xi \frac{1-\tan ^{2} p}{\tan ^{2} \xi-\tan ^{2} p} \\
& =2 y z \tan \xi \frac{(y+z)^{2}\left(1-\tan ^{2} p\right)}{(y+z)^{2} \tan ^{2} \xi-(y-z)^{2} \tan ^{2} \xi} \\
& =\frac{(y+z)^{2}-(y-z)^{2} \tan ^{2} \xi}{2 \tan ^{2}} .
\end{aligned}
$$

We may assume that $[A B C]=3 / 2$. Then we have

$$
\begin{aligned}
(y+z)^{2}-(y-z)^{2} \tan ^{2} \xi & =2 \tan \xi \\
\left(1-\tan ^{2} \xi\right)\left(y^{2}+z^{2}\right)+2 y z\left(1+\tan ^{2} \xi\right) & =2 \tan \xi \\
\left(y^{2}+z^{2}\right) \cos A+2 y z-\sin A & =0 .
\end{aligned}
$$

We record this in the following theorem.

Theorem 5. The trilinears $x: y: z$ of the equiareality center $\mathcal{E}$ of an acute triangle $A B C$ are defined by the equations

$$
\begin{aligned}
& \left(y^{2}+z^{2}\right) \cos A+2 y z-\sin A=0 \\
& \left(z^{2}+x^{2}\right) \cos B+2 z x-\sin B=0 \\
& \left(x^{2}+y^{2}\right) \cos C+2 x y-\sin C=0 .
\end{aligned}
$$




\section{Coincidence of $\mathcal{E}$ with the other traditional centers}

Let $A B C$ be a triangle and let $\mathcal{G}, \mathcal{O}, \mathcal{I}, \mathcal{H}$ denote its centroid, circumcenter, incenter, orthocenter, respectively. We shall show that $\mathcal{E}$ cannot coincide with any of these centers except when $A B C$ is equilateral. For $\mathcal{E}$ to be defined, we assume of course that $A B C$ is acute.

Note that to study the possible coincidence of $\mathcal{E}$ with a center whose trilinears $\left(x_{0}, y_{0}, z_{0}\right)$ are given, one needs only substitute $(x, y, z)=\lambda\left(x_{0}, y_{0}, z_{0}\right)$ in (4) and then solves the resulting equations. This may turn out to be difficult and clumsy. In this case, a purely geometric treatment would be desirable. Also, it may turn out that the $(\alpha: \beta: \gamma)$ coordinates (as defined in (1)) of a given center $P$ are easy to calculate. One then sets these equal to $(1: 1: 1)$ and solves the resulting equations. All of these approaches are illustrated below.

For the reader's convenience, we list below trilinears and barycentrics of the traditional centers. For these and anything that has to do with triangle centers, we refer the reader to [4] and [5].

\begin{tabular}{|c|c|c|}
\hline Center & Trilinears & Barycentrics \\
\hline Centroid $\mathcal{G}$ & $\left(\frac{1}{a}: \frac{1}{b}: \frac{1}{c}\right)$ & $(1: 1: 1)$ \\
\hline Circumcenter $\mathcal{O}$ & $(\cos A: \cos B: \cos C)$ & $(\sin 2 A: \sin 2 B: \sin 2 C)$ \\
\hline Incenter $\mathcal{I}$ & $(1: 1: 1)$ & $(a: b: c)=(\sin A: \sin B: \sin C)$ \\
\hline Orthocenter $\mathcal{H}$ & $(\sec A: \sec B: \sec C)$ & $(\tan A: \tan B: \tan C)$ \\
\hline
\end{tabular}

In the next theorems, we refer to (1) for the definitions of $\alpha, \beta, \gamma$.

Theorem 6. If $A B C$ is an acute triangle in which $\mathcal{E}=\mathcal{I}$ or $\mathcal{E}=\mathcal{O}$, then $A B C$ is equilateral.

Proof. Using the facts that

$$
\begin{aligned}
(\alpha(\mathcal{I}): \beta(\mathcal{I}): \gamma(\mathcal{I})) & =\left(\cot \frac{A}{2}: \cot \frac{B}{2}: \cot \frac{C}{2}\right), \\
(\alpha(\mathcal{O}): \beta(\mathcal{O}): \gamma(\mathcal{O})) & =(\sin 2 B+\sin 2 C: \sin 2 A+\sin 2 C: \sin 2 A+\sin 2 B), \\
(\alpha(\mathcal{E}): \beta(\mathcal{E}): \gamma(\mathcal{E})) & =(1: 1: 1),
\end{aligned}
$$

we conclude that

$$
\begin{aligned}
\mathcal{I}=\mathcal{E} & \Longrightarrow \cot \frac{A}{2}=\cot \frac{B}{2}=\cot \frac{C}{2} \Longrightarrow A=B=C \\
\mathcal{O}=\mathcal{E} & \Longrightarrow \sin 2 B+\sin 2 C=\sin 2 C+\sin 2 A=\sin 2 A+\sin 2 B \\
& \Longrightarrow \sin 2 A=\sin 2 B=\sin 2 C \Longrightarrow A=B=C
\end{aligned}
$$

The last implication follows from the fact that the possibility $2 B+2 C=\pi$ leads to $A=\pi / 2$, contradicting the assumption that $A B C$ is acute. This will be freely used later. 
Theorem 7. If $A B C$ is an acute triangle in which $\mathcal{E}=\mathcal{G}$, then $A B C$ is equilateral.

Proof. It is obvious that the medians $A A^{\prime}, B B^{\prime}, C C^{\prime}$ of $A B C$ divide $A B C$ into six triangles of equal areas; see [1, Theorem 5]. Therefore the quadrilaterals $\mathcal{G} C^{\prime} A B^{\prime}, \mathcal{G} A^{\prime} B C^{\prime}$, $\mathcal{G} B^{\prime} C A^{\prime}$ have equal areas. Also, the orthocenter $\mathcal{H}$ of $A B C$ must lie in one of these quadrilaterals, say in $\mathcal{G} B^{\prime} C A^{\prime}$; see Fig. 7. Then

$$
\angle B B^{\prime} C \leq \frac{\pi}{2}, \quad \angle A A^{\prime} C \leq \frac{\pi}{2} .
$$

Hence the orthogonal projections $J, I$ of $\mathcal{G}$ onto the sides $C A, C B$, respectively, lie in the quadrilateral $\mathcal{G} B^{\prime} C A^{\prime}$, and thus $\mathcal{G} I C J$ lies inside $\mathcal{G} B^{\prime} C A^{\prime}$. If $\mathcal{E}=\mathcal{G}$, then these quadrilaterals have the same area and hence $B^{\prime}=J$ and $A^{\prime}=I$. Thus the medians $B B^{\prime}$, $A A^{\prime}$ are perpendicular to the respective sides and the triangle is equilateral, as claimed.

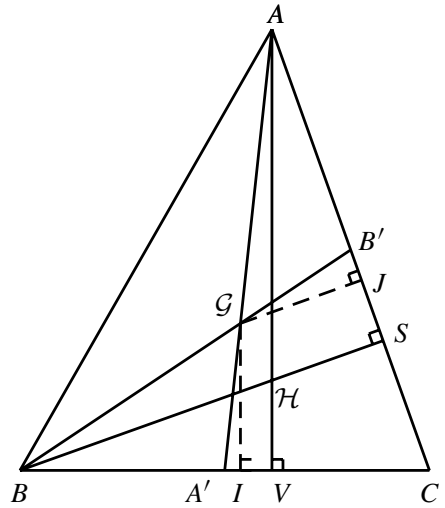

Fig. 7

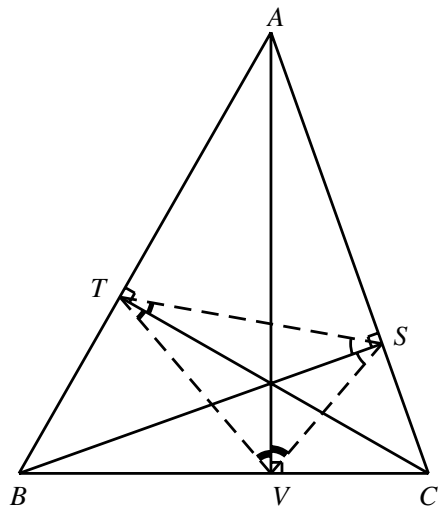

Fig. 8

Theorem 8. If $A B C$ is an acute triangle in which $\mathcal{E}=\mathcal{H}$, then $A B C$ is equilateral.

Proof. Let $A V, B S, C T$ be the altitudes of $A B C$ (necessarily through $\mathcal{H}$ ); see Fig. 8 . Clearly the triangles $C S V$ and $C B A$ are similar with similarity ratio $C S / C B=\cos C$. Therefore $[C S V] /[C B A]=\cos ^{2} C$. Similarly, $[B V T] /[C B A]=\cos ^{2} B$. Hence

$$
\frac{[C S V]}{[B V T]}=\frac{\cos ^{2} C}{\cos ^{2} B}
$$

Also, it follows from the cyclicity of the quadrilaterals $A T \mathcal{H} S, B V \mathcal{H} T, C S \mathcal{H} V$ that the angles of $V S T$ are given by

$$
V=\pi-2 A, \quad S=\pi-2 B, \quad T=\pi-2 C,
$$

and that $A V, B S, C T$ are the angle bisectors of the triangle $V S T$. Therefore

$$
\frac{[V S \mathcal{H}]}{[V T \mathcal{H}]}=\frac{(V S)(V \mathcal{H}) \sin \angle \mathcal{H} V S}{(V T)(V \mathcal{H}) \sin \angle \mathcal{H} V T}=\frac{V S}{V T}=\frac{\sin T}{\sin S}=\frac{\sin (\pi-2 C)}{\sin (\pi-2 B)}=\frac{\sin 2 C}{\sin 2 B}
$$


Note also that

$$
\sin 2 C-\sin 2 B=2 \cos (C+B) \sin (C-B)=-2 \cos A \sin (C-B) .
$$

Suppose now that $C>B$. Then it follows from (8) and (9) that $[V S \mathcal{H}]<[V T \mathcal{H}]$. It also follows from (6) that $[C S V]<[B V T]$. Adding, we obtain $[C S \mathcal{H} V]<[B V \mathcal{H} T]$. Thus we have proved that

$$
C>B \Longrightarrow \gamma(\mathcal{H})<\beta(\mathcal{H})
$$

Suppose now that $\mathcal{E}=\mathcal{H}$. Then $\gamma(\mathcal{H})=\beta(\mathcal{H})$ and hence $C=B$. Similarly $B=A$ and $A B C$ is equilateral.

Remark 9. It is well-known that if any two of the centers $\mathcal{G}, \mathcal{I}, \mathcal{O}, \mathcal{H}$ of a triangle coincide, then the triangle is equilateral; see [2] and the references therein. It also goes without saying that all centers of an equilateral triangle coincide.

In view of Remark 9 above, Theorems 6, 7, 8 can be summarized in the next theorem.

Theorem 10. An acute triangle is equilateral if and only if two (and hence all) of the centers $\mathcal{E}, \mathcal{G}, \mathcal{I}, \mathcal{O}, \mathcal{H}$ coincide.

\section{Another related center}

Let $A B C$ be an acute triangle. For $X$ on $B C$, let $X Y, X Z$ be the perpendiculars from $X$ dropped onto the sides $A C, A B$, respectively. As $X$ moves from $B$ to $C,[X Z B] /[X Y C]$ increases from 0 to $\infty$. Thus there exists a unique point, to be denoted by $A^{\prime}$, for which $[X Z B]=[X Y C]$. We define $B^{\prime}, C^{\prime}$ similarly.

Theorem 11. The cevians $A A^{\prime}, B B^{\prime}, C C^{\prime}$ are concurrent. The barycentrics of the point of concurrence are given by

$$
\sqrt{\sin 2 A}: \sqrt{\sin 2 B}: \sqrt{\sin 2 C},
$$

and the trilinears are given by

$$
\sqrt{\cot A}: \sqrt{\cot B}: \sqrt{\cot C} \text {. }
$$

Proof. Observe that if $X$ lies on $B C$ and if $t=C A^{\prime}$, as shown in Fig. 1, then the condition $[X Z B]=[X Y C]$ is equivalent to $(a-t)^{2} \cos B \sin B=t^{2} \cos C \sin C$, i.e., $(a-t)^{2}$ : $t^{2}=\sin 2 C: \sin 2 B$. Concurrence of $A A^{\prime}, B B^{\prime}, C C^{\prime}$ follows immediately from Ceva's theorem. It also follows that if $P$ is the point of concurrence, then $[P A B]:[P A C]=$ $(a-t): t=\sqrt{\sin 2 C}: \sqrt{\sin 2 B}$. Hence the barycentrics of $P$ are as given in (11). The statement about the trilinears follows from

$$
\frac{\sqrt{\sin 2 A}}{a} \sim \frac{\sqrt{\sin 2 A}}{\sin A}=\sqrt{\frac{2 \sin A \cos A}{\sin ^{2} A}} \sim \sqrt{\cot A} .
$$


Definition 12. The point of concurrence will be denoted by $\mathcal{E}_{0}$.

Theorem 13. An acute triangle is equilateral if and only if two (and hence all) of the centers $\mathcal{E}, \mathcal{E}_{0}, \mathcal{G}, \mathcal{I}, \mathcal{O}, \mathcal{H}$ coincide.

Proof. In view of Remark 9 above and in view of the symmetry, it is sufficient to show that each of the assumptions $\mathcal{E}_{0}=\mathcal{G}, \mathcal{E}_{0}=\mathcal{O}, \mathcal{E}_{0}=\mathcal{I}, \mathcal{E}_{0}=\mathcal{H}, \mathcal{E}_{0}=\mathcal{E}$ leads to $A=B$. But

$$
\begin{aligned}
\mathcal{E}_{0}=\mathcal{G} & \Longrightarrow \sqrt{\sin 2 A}=\sqrt{\sin 2 B} \Longrightarrow A=B, \\
\mathcal{E}_{0}=\mathcal{O} & \Longrightarrow \frac{\sin 2 A}{\sqrt{\sin 2 A}}=\frac{\sin 2 B}{\sqrt{\sin 2 B}} \Longrightarrow \sin 2 A=\sin 2 B \quad \Longrightarrow \quad A=B, \\
\mathcal{E}_{0}=\mathcal{I} & \Longrightarrow \frac{\sin A}{\sqrt{\sin 2 A}}=\frac{\sin B}{\sqrt{\sin 2 B}} \Longrightarrow \tan A=\tan B \quad \Longrightarrow A=B, \\
\mathcal{E}_{0}=\mathcal{H} & \Longrightarrow \frac{\tan A}{\sqrt{\sin 2 A}}=\frac{\tan B}{\sqrt{\sin 2 B}} \Longrightarrow \frac{\sin A}{\cos ^{3} A}=\frac{\sin B}{\cos ^{3} B} \\
& \Longrightarrow \tan A\left(1+\tan ^{2} A\right)=\tan B\left(1+\tan ^{2} B\right) \\
& \Longrightarrow A=B, \text { because } x\left(1+x^{2}\right) \text { is increasing. }
\end{aligned}
$$

It remains to deal with the case $\mathcal{E}=\mathcal{E}_{0}$. If $\mathcal{E}=\mathcal{E}_{0}$, then a multiple of the trilinears of $\mathcal{E}_{0}$ given in (12) must satisfy the equations (4) for the trilinears of $\mathcal{E}$. Substituting $(x, y, z)=$ $\lambda(\sqrt{\cot A}, \sqrt{\cot B}, \sqrt{\cot C})$ in the first equation in (4), we obtain

$$
\lambda^{2}\left(\frac{\cos B}{\sin B}+\frac{\cos C}{\sin C}\right) \cos A+2 \lambda^{2} \sqrt{\frac{\cos B \cos C}{\sin B \sin C}}=\sin A .
$$

Multiplying by $(\sin B \sin C) / \lambda^{2}$ and using the identity $\cos B \sin C+\sin B \cos C=\sin (B+$ $C)=\sin A$, we obtain

$$
\sin A \cos A+2 \sqrt{\sin B \cos B \sin C \cos C}=\frac{\sin A \sin B \sin C}{\lambda^{2}} .
$$

Using the double angle formula, we obtain

$$
\sin 2 A+2 \sqrt{\sin 2 B \sin 2 C}=\frac{2 \sin A \sin B \sin C}{\lambda^{2}} .
$$

Using the other two equations in (4), and letting

$$
u=\sqrt{\sin 2 A}, \quad v=\sqrt{\sin 2 B}, \quad w=\sqrt{\sin 2 C},
$$

we obtain

$$
u^{2}+2 v w=v^{2}+2 w u=w^{2}+2 u v
$$

Therefore

$$
(u-v)(u+v-2 w)=(v-w)(v+w-2 u)=(w-u)(w+u-2 v)=0 .
$$


If no two of $u, v, w$ are equal, then $u+v-2 w=v+w-2 u=0$, and hence $u+v+w=$ $3 w=3 u$, and the contradiction $w=u$. If exactly two of $u, v, w$ are equal, say $u=v \neq w$, then $v+w-2 u=0$ and hence the contradiction $w=u$. We are left with the possibility $u=v=w$, i.e., $\sin 2 A=\sin 2 B=\sin 2 C$. Therefore $A=B=C$.

\section{The obtuse case}

If $A B C$ is an obtuse triangle and if $P$ is a point inside $A B C$, then the perpendiculars $P X$, $P Y, P Z$ from $P$ to the sides $B C, C A, A B$ do not necessarily fall onto the sides; see Fig. 9 . In this case, parts of the quadrilaterals $P Y A Z, P Z B X, P X C Y$ fall outside the triangle, and some of these quadrilaterals are self crossing; see Fig. 9. It would be interesting to

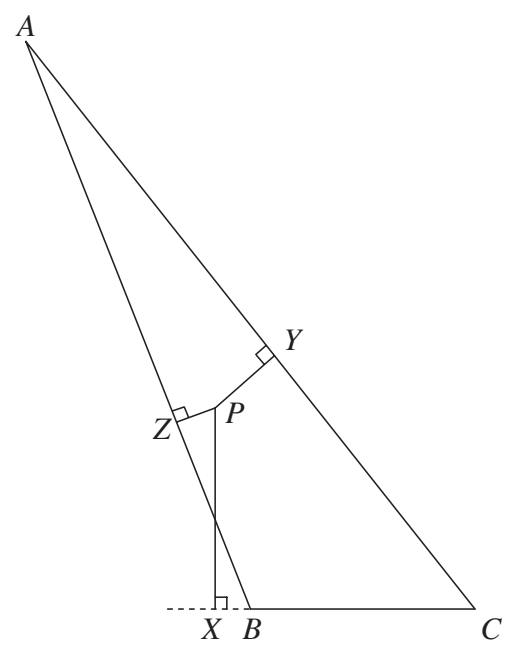

Fig. 9

explore possibilities of assigning areas to such quadrilaterals in such a way that the sum of the areas of $P Y A Z, P Z B X, P X C Y$ is still equal to that of $A B C$. One would also like to do the same for points outside $A B C$. Then one would investigate the existence and uniqueness of a point $P$, inside or outside $A B C$, for which the areas of $P Y A Z, P Z B X$, $P X C Y$ are equal. Calling such a point an equiareality point, one may try to characterize those obtuse triangles that have an interior equiareality point, and those obtuse triangles that have an interior equiareality point $P$ for which the three associated quadrilaterals lie inside $A B C$.

\section{Acknowledgements}

The first named author is supported by a research grant from Yarmouk University. 


\section{References}

[1] Abu-Saymeh, S.; Hajja, M.: In search of more triangle centers - A source of classroom projects in Euclidean geometry. Internat. J. Math. Ed. Sci. Tech. 36 (2005), 889-912.

[2] Hajja, M.: Triangle centers - Some questions in Euclidean geometry. Internat. J. Math. Ed. Sci. Tech. 32 (2001), 21-37.

[3] Hajja, M.; Krasopoulos, P.T.: Problem Proposal. Aufgabe 1275, El. Math. 65, No. 1 (2010), 37.

[4] Kimberling, C.: Triangle centers and central triangles. Congr. Numer. 129 (1998), 1-285.

[5] Kimberling, C.: Encyclopedia of Triangle Centers. http://faculty.evansville.edu/ck6/encyclopedia/

Mowaffaq Hajja

Mathematics Department

Yarmouk University

Irbid, Jordan

e-mail:mhajja@yu.edu.jo

mowhajja@yahoo.com

Panagiotis T. Krasopoulos

Social Insurance Institute

General Administration of Informatics

Papadiamantopoulou 87

11527 Athens, Greece

e-mail: pankras@in.gr 